PHYSICAL REVIEW D 93, 119903(E) (2016)

\title{
Erratum: Single-inclusive production of hadrons and jets \\ in lepton-nucleon scattering at NLO \\ [Phys. Rev. D 92, 014001 (2015)]
}

\section{Patriz Hinderer, Marc Schlegel, and Werner Vogelsang}

(Received 3 June 2016; published 17 June 2016)

DOI: 10.1103/PhysRevD.93.119903

The following corrections apply to the numerical results shown in this article:

(1) Figures 5(a) and 6(a) show the differential cross section $\frac{\mathrm{d} \sigma}{\mathrm{d} x_{F} \mathrm{~d} P_{h \perp}}$, multiplied by the kinematical factor $\left(x_{F}^{2}+x_{T}^{2}\right)$. The cross section shown in Fig. 5(a) has furthermore been averaged over the bin $1 \mathrm{GeV} \leq P_{h \perp} \leq 2.2 \mathrm{GeV}$ in hadron transverse momentum.

(2) The cross sections vs $P_{h \perp}$ shown in Figs. 5(b) and 6(b) have been averaged over hadron rapidity $\eta$ rather than Feynman $x_{F}$. The bin limits in $\eta$ were computed using the ones given for $x_{F}$ and the relation $x_{F}=x_{T} \sinh (\eta)$.

(3) The results shown in Figs. 7(a) and 7(b) were obtained for $\sqrt{S}=18 \mathrm{GeV}$ rather than $\sqrt{S}=17.4 \mathrm{GeV}$ and for electron scattering instead of muon scattering as would be appropriate for COMPASS. The corrected plots are shown below.
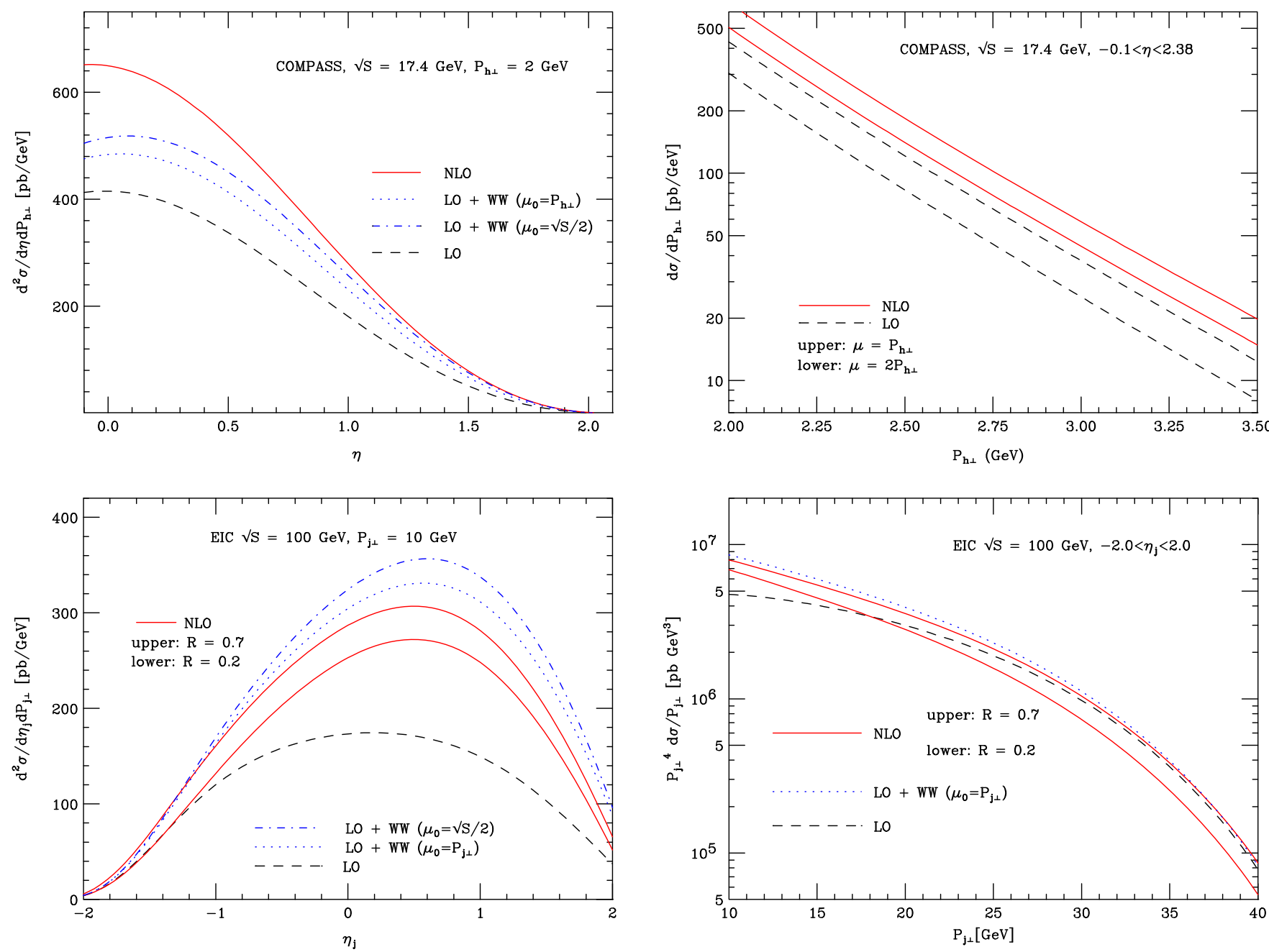

Corrected versions of Figs. 7(a) (upper panel, left) and 7(b) (upper panel, right), and Figs. 9(a) (lower panel, left) and 9(b) (lower panel, right). 
(4) Figure 8(a) was obtained for a fixed transverse momentum $P_{h \perp}=10 \mathrm{GeV}$ instead of $2 \mathrm{GeV}$ as incorrectly indicated in the caption. Furthermore, the cross sections in Figs. 8(b) and 9(b) have been averaged (rather than integrated) over hadron rapidity.

(5) We fixed an error in the code used to produce the plots in Figs. 9(a) and (b). The corrected plots are shown below. We note that none of these corrections alters the main conclusions of our paper, since only the overall normalization of the plots is affected. Finally, we also note that a factor of $4 \pi$ is missing on the right-hand-side of the Weizsäcker-Williams contributions in Eq. (29). This mistake, however, did not propagate into our numerical calculations.

We thank Umberto d'Alesio for pointing out to us the inconsistencies in our results. 